\title{
Quantifying Gait Similarity: User Authentication and Real-World Challenge
}

\author{
Marc Bächlin, Johannes Schumm, Daniel Roggen, and Gerhard Töster \\ Wearable Computing Laboratory, ETH Zürich, Switzerland \\ \{baechlin, schumm, roggen, troester\}@ife.ee.ethz.ch \\ http://www.ife.ee.ethz.ch
}

\begin{abstract}
Template-based approaches using acceleration signals have been proposed for gait-based biometric authentication. In daily life a number of real-world factors affect the users' gait and we investigate their effects on authentication performance. We analyze the effect of walking speed, different shoes, extra load, and the natural variation over days on the gait. Therefore we introduce a statistical Measure of Similarity (MOS) suited for template-based pattern recognition. The MOS and actual authentication show that these factors may affect the gait of an individual at a level comparable to the variations between individuals. A change in walking speed of $1 \mathrm{~km} / \mathrm{h}$ for example has the same MOS of $20 \%$ as the in-between individuals' MOS. This limits the applicability of gait-based authentication approaches. We identify how these real-world factors may be compensated and we discuss the opportunities for gaitbased context-awareness in wearable computing systems.
\end{abstract}

\section{Introduction}

Biometric authentication is the process of verifying the claimed identity of an individual by his or her physiological or behavioral characteristics. Characteristics include e.g. fingerprint, iris, retina, face, voice, handwriting. This paper presents results on gait-based biometrics. Most of the previous work in gait based user authentication is based on machine vision techniques e.g. 7/11. An inherent advantage of vision-based gait recognition systems is to capture the gait of a person from a distance. Another, relative new direction, is the user authentication based on miniature acceleration sensors to capture the gait profile. Gait-based authentication using accelerometer sensor was demonstrated in [1|4|9|10] using template-based approaches. Primary advantage of on-body biometric authentication systems is that it excludes the need of ambient technology. Potential applications include smart clothes/bags, or electronic devices that are able to detect who is carrying them to adapt their functionality, or that can detect and report that they were stolen.

Intrinsic gait variability is challenging in authentication systems. For accelerometer based authentication systems these intrinsic gait variability has not been analyzed over long periods of time, nor when parameters typical for realworld scenarios are varied (such as e.g. which pair of shoes is worn or how much

M. Tistarelli and M.S. Nixon (Eds.): ICB 2009, LNCS 5558, pp. 1040-1049, 2009.

(C) Springer-Verlag Berlin Heidelberg 2009 
weight is carried). In order to characterize gait in real-world scenarios we introduce a method to statistically quantify similarity between gait data sets. We use this method to characterize gait variability on a dataset composed of 5 subjects, recorded on 5 consecutive days. This data set includes variations in walking speed, weight carried and shoes worn. We report the results and analyze the implications for gait-based authentication.

\section{Related Work}

Identifying users from gait patterns using accelerometers is based on the assumption that the gait acceleration profile ("template") is unique to some extent for every person. During training, a feature template vector that represents characteristics of the gait of the person to authenticate is computed. The same feature vector is computed during the authentication process and compared to the feature template. The feature vector components commonly used are coming from the temporal domain (e.g. acceleration profile before/after the heel strike), and frequency domain (e.g. power spectrum of a footstep). Usually multiple feature vectors are available (e.g. when the feature vectors are derived from individual steps or out of a sliding window), and the feature template is the average of the feature vectors.

Ailisto and Mäntyjärvi et al. 119] were the first to report on acceleration sensor based gait authentication. They recorded the acceleration of the waist of 36 subjects on two different days while the subjects were walking $20 \mathrm{~m}$ at their normal, slow and fast walking speed. The performances of three methods were analyzed: (i) a correlation method using a step template in the time domain; (ii) a frequency-domain method using the first 40 FFT coefficients as feature template in the frequency domain; (iii) a data distribution statistics, using the data distribution as feature vector. Using the correlation method they achieved best results with an Equal Error Ratio (EER) of 7\%. Further work supported their results. Gafurov et al. 56] recorded the acceleration of the right ankle of 21 subjects with a $16 \mathrm{~Hz}$ sampling frequency during a $70 \mathrm{~m}$ walk. They achieved an EER of $9 \%$ and $5 \%$ respectively. Gafurov and Buvarp [3] further showed that it is not possible for a person to imitate the gait of another person. A combination of a sensor-based gait and voice biometric has been proposed by Vildjiounaite et al. 12. The sensor fusion increases the performance of the recognition system in a noisy or loud environment. Liu Rong et al. [10] recorded 5 acceleration data sets at the hip of 35 participants. With a dynamic time warping (DTW) method to normalize the step lengths they achieved an EER of $6,7 \%$.

The literature differs in the number of acceleration signals that are used as well as the sensor positions, however no reported results clearly outperforms the others. The common points of all presented works are short recordings, no variability and the use of template based matching or comparison techniques. However, in real-world scenarios a number of parameters may affect gait, including: walking speed, shoe type, ground, drunkenness and injuries. The effects of these parameters has not been studied, despite their likely influence on "feature templates" and thus on the authentication accuracy. 


\section{$3 \quad$ Statistical Template Similarity Measure}

The authentication performance depends on how accurately a feature template represents the actual feature vectors extracted from the underlying signal. Performance also depends on how dissimilar are the feature vectors of two different datasets. In order to objectively assess this (dis)similarity we devise a statistical measure of similarity. This measure is (i) generic; (ii) classifier independent; (iii) and it reveals specific locations in the feature vector with high entropy.
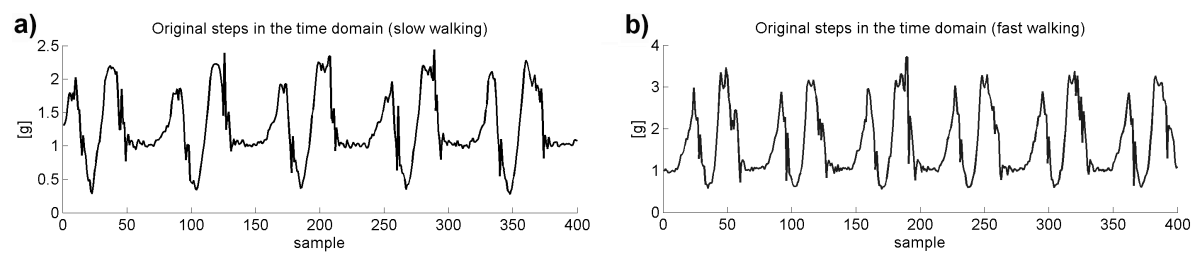

Fig. 1. Two acceleration signal examples: a) $4 \mathrm{~km} / \mathrm{h}$ walking b) $6 \mathrm{~km} / \mathrm{h}$ walking

We measure the gait acceleration at $64 \mathrm{~Hz}$ with a three-axis MEMS acceleration sensor placed at the ankle (see 2] for sensor details). This three-axis sensor gives an acceleration vector $\boldsymbol{n}=(x, y, z)$ with following axis orientation: $x=$ horizontal forward, $y=$ vertical, $z=$ horizontal lateral. In figure 1 we show the norm of the acceleration signal with walking speeds $4 \mathrm{~km} / \mathrm{h}$ and $6 \mathrm{~km} / \mathrm{h}$. The signal is then converted into a feature vector that encores characteristics of the gait style. We compare four feature types (two in the time domain and two in the frequency domain) applied to 3 sensor axis combinations (signal types).

Feature type A: A window of 64 samples is aligned on the heel strike. Each sample in the window is one component of the feature vector, yielding a feature vector with 64 components. This feature vector is sensitive to walking speed.

Feature type B: In order to be independent of walking speed, the step length is normalized by dynamic time warping (DTW) to 64 samples. The acceleration magnitudes are normalized to values between 0 and 1 [10]. After the normalization all steps have the same length and amplitude (see figure 2). The normalized acceleration samples form the feature vector.

Feature type C: A jumping window of length 256 samples and jump length of 64 samples is shifted over the signal. A 256 point FFT is computed on the window and the lower $40 \mathrm{FFT}$ coefficients form the feature vector.

Feature type $\mathbf{D}$ is identical to feature type $\mathrm{C}$ except that the window is centered on the heel strike rather than placed at regular intervals.

Signal type a: The features are computed on the acceleration norm $|n|=$ $\sqrt{x^{2}+y^{2}+z^{2}}$. 
a)

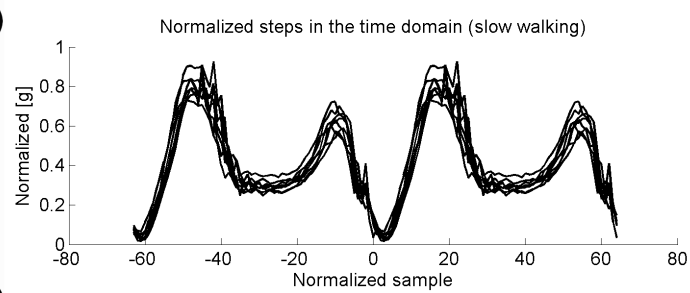

b)

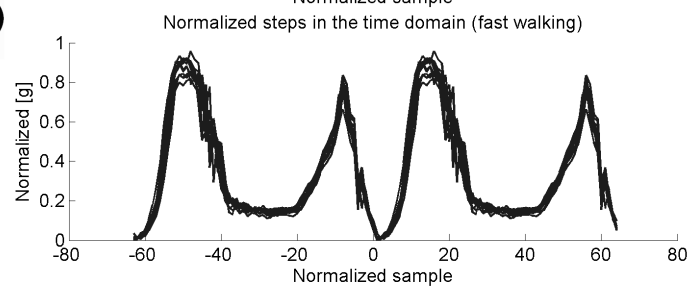

Fig. 2. Normalized steps superimposed on each other a) Normalized steps of $4 \mathrm{~km} / \mathrm{h}$ walking b) Normalized steps of $6 \mathrm{~km} / \mathrm{h}$ walking

Signal type b: The features are computed on the vertical axis of the acceleration.

Signal type c: The features are computed on two signals: the vertical and forward/backward acceleration axes. The feature vector is twice the size as with signal type a or b. The first half are features derived from the vertical acceleration; the second half are features derived from the forward/backward acceleration.

The combination of 4 feature types and 3 signal types results in 12 kinds of feature vectors. We denote the feature vector kind by Feature signal $_{\text {with }}$ Feature $\in\{A, B, C, D\}$ and signal $\in\{a, b, c\}$. For each window, the feature vector is computed. Statistical quantification of the similarity between the multiple feature vectors extracted from two datasets is described below. For authentication, multiple feature vectors are averaged into a single feature template over a recording period.

Assume two sets $\boldsymbol{F}_{X}$ and $\boldsymbol{F}_{Y}$ of $N$ feature vectors of length $L$ computed on datasets $S_{X}$ and $S_{Y}: \boldsymbol{F}_{X}^{i}=\left[f_{X, 0}^{i}, \ldots, f_{X, L-1}^{i}\right]$ and $\boldsymbol{F}_{Y}^{i}=\left[f_{Y, 0}^{i}, \ldots, f_{Y, L-1}^{i}\right]$, with $i=0 \ldots N-1$. We introduce a statistical measure of similarity between feature vector set $\boldsymbol{F}_{X}$ and $\boldsymbol{F}_{Y}$. For each feature $l=0,1, \ldots, L-1$ we compute the one-way analysis of variance (ANOVA) between datasets $S_{X}$ and $S_{Y}$, i.e. $p_{l}=\operatorname{Anova}\left(\left\{f_{X, l}^{0}, \ldots, f_{X, l}^{N-1}\right\} ;\left\{f_{Y, l}^{0}, \ldots, f_{Y, l}^{N-1}\right\}\right) . p_{l}$ is the p-value under the null hypothesis that all data samples at position $l$ in the feature vector are drawn from populations with the same mean. If $p_{l}$ is near zero, it suggests that one sample mean is significantly different than the other sample mean.

For example, with the feature type B, a feature vector that represents the gait acceleration profile is generated at each heel strike (figure 2). Values near zero in $\boldsymbol{p}=\left[p_{0}, \ldots, p_{L-1}\right]$ indicate that the acceleration value at position $l$ in the feature vector is statistically different between the gait datasets $S_{X}$ and $S_{Y}$. As feature types $\mathrm{C}$ and $\mathrm{D}$ are composed in the frequency domain, $\boldsymbol{p}$ indicates statistical similarity of the frequency components. 
In order to obtain a single valued statistical similarity measure between the feature vectors of dataset $S_{X}$ and $S_{Y}$, we define the Measure Of Similarity (MOS) value as the percentage of positions in the feature vector that are not statistically significantly different $(p>0.05)$. The MOS represents the similarity of two feature sets, and 1-MOS represents the dissimilarity of two feature sets. It allows to statistically compare different gait datasets when real-world factors are varied.

\section{Experiment}

We analyze the effect of shoes, weight and time (day to day changes) on gait; we compare that to the differences in gait styles from persons to persons. Concretely we compare the set of feature vectors obtained from one recording to the set obtained from another recording using the MOS.

Data is recorded while subjects walk on a treadmill. This allows to control the walking speed and ensures that the behavior of participants is similar among themselves and from one day to the next. The participants performed 8 walking tasks on 5 days over 2 weeks. The 8 walking tasks are walking with a main pair of shoes at different speeds $(3,4,5$ and $6 \mathrm{~km} / \mathrm{h})$, then at $4 \mathrm{~km} / \mathrm{h}$ with additional weight (backpack with 6 and $10 \mathrm{~kg}$ ) as well as without shoes and with an alternate pair of shoes. Each task lasts at least $1 \mathrm{~min}$ every day. This results in $8 \mathrm{~min}$ walking data per day and participant. 5 male subjects with age between 25-28 years, weight between $65-75 \mathrm{~kg}$ and height between $1.75-1.85 \mathrm{~m}$ participated in the experiments, resulting in overall $200 \mathrm{~min}$ (about 12000 steps) of walking data.

\section{$5 \quad$ Results and Discussion}

In figure 3 we present the results of the statistical similarity analysis between gait datasets using feature type $A_{a}$ (steps in the time domain, without normalization using the norm of all axes). The bars are the MOS value, the lines indicate the standard deviation (std) over all used data sets.

Figure 3a) depicts the results of the statistical similarity analysis between the first $30 \mathrm{sec}$ and the second $30 \mathrm{sec}$ of each $1 \mathrm{~min}$ data set. The standard deviation reflects the variation between the tasks and days. This is a baseline measure that indicates the highest MOS one is likely to expect (about $80 \%$ ). It represents the intrinsic gait similarity (or variability) typical for human walking. Figure $3 \mathrm{~b}$ ) shows that the MOS is about $20 \%$ for two different subjects doing the same task. As expected the intrinsic gait similarity of one subject is higher than the similarity between subjects. Thus, at identical tasks different persons have different gait patterns that can be used for authentication.

However, the tasks affect the gait. Figure 3 $1 \mathrm{c}$ ) shows the influence of changing weight $(+6,+10 \mathrm{~kg})$, kind of shoes (shoes) as well as speed $(-1,+1,+2 \mathrm{~km})$. The additional weight has the smallest influence on the gait signal. Changing or removing shoes reduces the MOS by more than $50 \%$. A change of speed as small 
a)

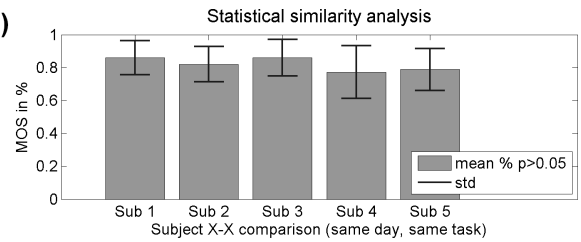

b)

Statistical similarity analysis

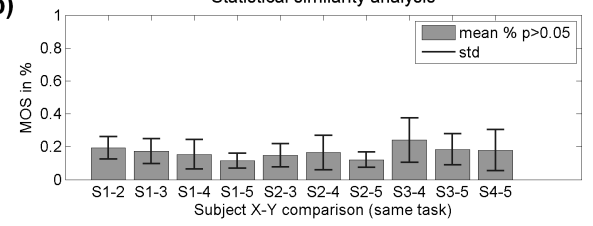

c)

Statistical similarity analysis

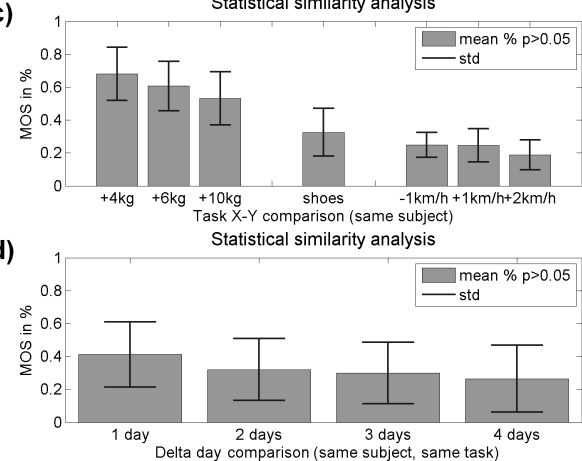

Fig. 3. MOS values with feature type Aa: a) baseline measure b) between different subjects doing the same task c) between different tasks performed by the same subject d) between different days with identical task performed by the same subject

as $\pm 1 \mathrm{~km} / \mathrm{h}$ reduces the MOS to about $25 \%$. A change of $+2 \mathrm{~km} / \mathrm{h}$ reduces the MOS to about $20 \%$ that is comparable to the MOS between persons. Given that speed modifies the gait of one person (fig 3 c) in a range similar to what the gait of two persons at identical speeds differs (fig 3b) - seen by the similar MOS values - means that it is challenging to distinguish the two cases.

Figure 3d) shows the MOS of datasets taken on different days, with 1 to 4 days between the recordings. Surprisingly there is a low level of similarity between different days and the MOS tends to decrease with increasing time distance between the datasets. Future work is needed to assess whether there is a natural tendency for gait patterns to change over time, whether this is cyclic, or whether this may be caused by the user getting used to walk on the treadmill. Analysis of longer recordings will clarify this.

Results obtained using other feature kinds in the time domain show similar results to those presented in figure 3 . On the left side of figure 4 we show the result of the similarity analysis in the time domain using feature type $B_{a}$ (normalized steps using the acceleration norm). The normalization in time domain slightly improves the similarity measure of the intrinsic gait similarity (fig. $4 \mathrm{a}_{1}$ ), and minimizes the effect of weight (fig. $4 \mathrm{c}_{1}$ ), whereas other MOS values are similar to 

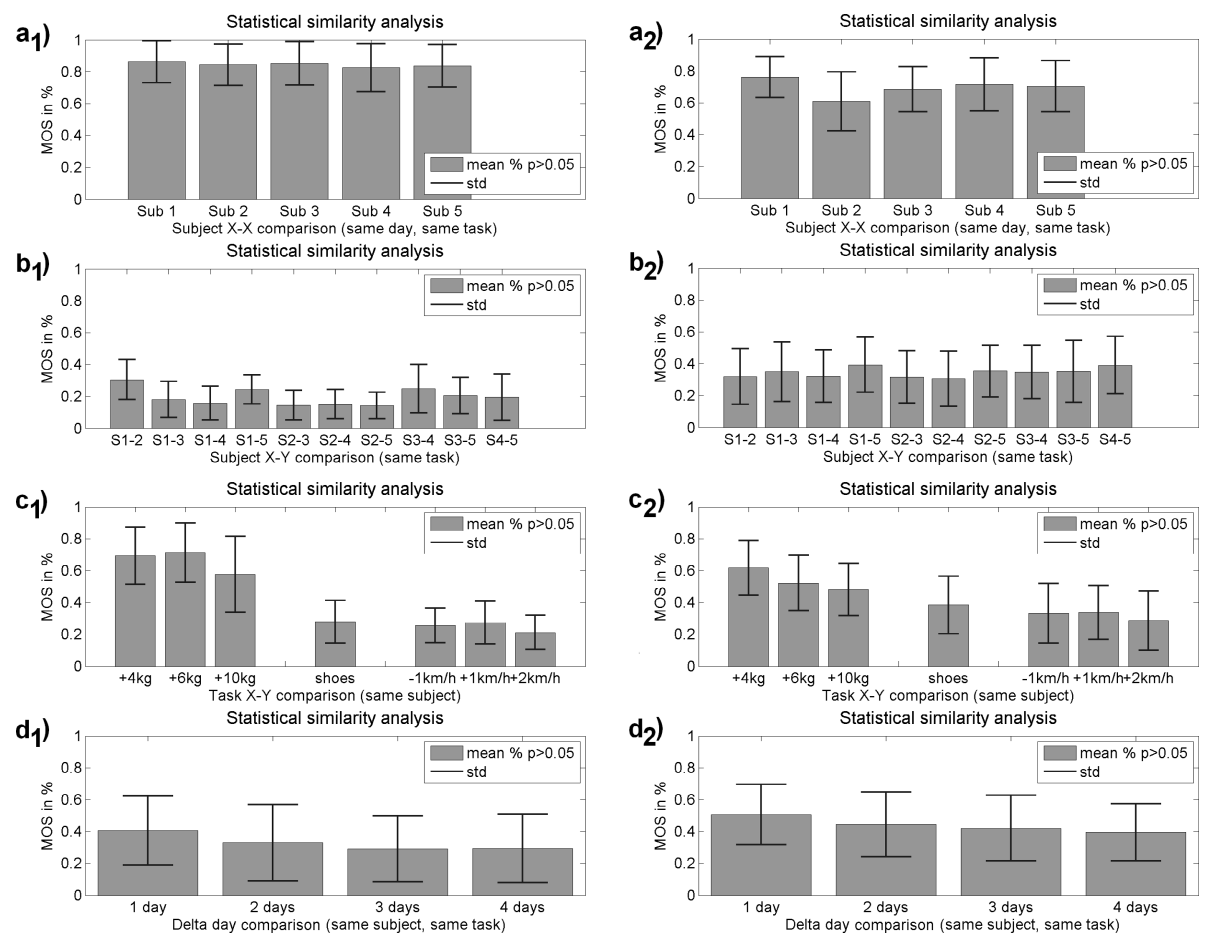

Fig. 4. Results of the statistical similarity analysis: Left side: using feature type $B a$ (Normalized steps using the norm); Right side: using feature type Da (step triggered FFT using the norm)

those obtained with feature type $A_{a}$. Despite time and amplitude normalization change in speed leads to MOS values in a range similar to those obtained with feature type $A_{a}$. This means that a change in speed does not only affect gait frequency or amplitude but changes the nature of the gait acceleration profile itself. This is visible in fig. 2, although acceleration peaks are aligned and of similar amplitude, the acceleration profile nevertheless differs. Similarly changes in shoes also affects the acceleration profile, not only its amplitude.

On the right side of figure 4 we show the result with frequency domain features $D_{a}$. Compared to previous results, the intrinsic gait similarity is lower, while the similarity between subjects is greater (fig $4 \mathrm{~b}_{2}$ ). Therefore this feature kind is less discriminative than the time domain features. The influence of weight, shoes and speed and variations over time on the MOS is lesser though than with time domain features (higher MOS) (fig $\left.4 \mathrm{c}_{2}, \mathrm{~d}_{2}\right)$. However overall trends are comparable. Results with other frequency domain features are similar.

To verify the link between the MOS values and authentication performance we apply the authentication scheme described in 310 on our dataset. It distinguishes an impostor dataset from a genuine dataset. A reference feature template $T_{X, D, T}^{R}$ is obtained from the person to authenticate $X$. It is computed on the first half of the $1 \mathrm{~min}$ gait recording. The reference template is the average 


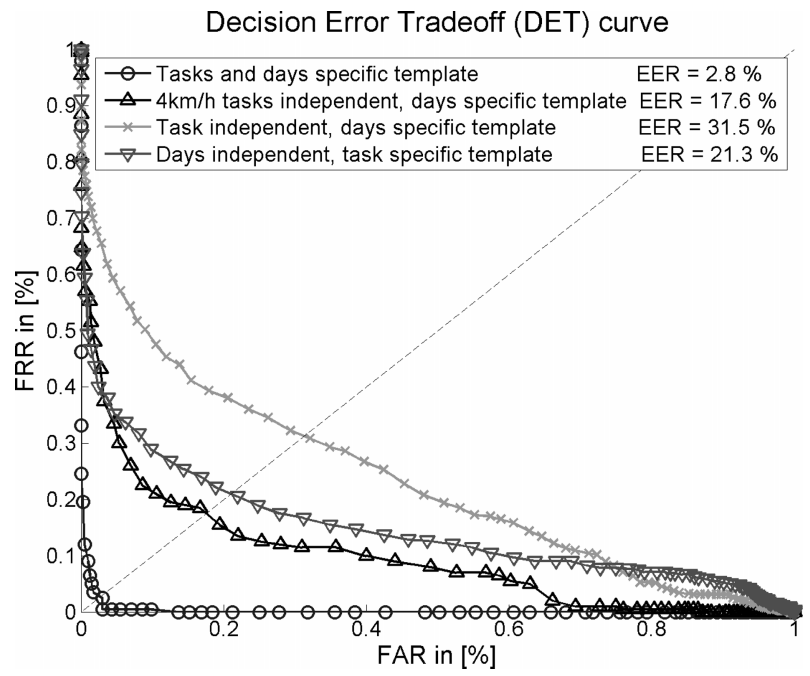

Fig. 5. Person authentication DEF curve for different data set combinations

feature vectors computed on the recording of a single task $T$ on a given day $D$ (task/day-dependent authentication) or the average of feature vectors computed on recordings of multiple tasks $T=t_{1} . . t_{n}$ or on multiple days $D=d_{1} . . d_{n}$ (task/day-independent authentication). A genuine $T_{X, D, T}^{G}$ template is obtained from the same person at a later timepoint. It is used to assess the system acceptation performance. It is computed on the second half of the gait recording of each task. An impostor template $T_{Y, D, T}^{I}$ is obtained from the gait of another person $Y$. It is used to assess the system rejection performance. The authentication scheme compares the normalized Euclidian distance between the genuine/impostor templates and the reference templates. It makes a decision (acceptation/rejection) based on a distance threshold that controls the tradeoff between False Acceptance Ratio (FAR) and False Rejection Ratio (FRR). This is represented by a Decision Error Tradeoff (DET) curve (see figure 5). The Equal Error Rate is the point in the curve where the FAR is equal to the FRR (lower EER is better). We denote the DET curve by $\operatorname{DET}\left(T_{X, D, T}^{R}, T_{X, D^{\prime}, T^{\prime \prime}}^{G}, T_{Y, D^{\prime \prime}, T^{\prime \prime}}^{I}\right)$. Unless specified below, it is computed and averaged on all combinations of 5 persons $X=x_{1} \ldots x_{5}$, $Y=y_{1} . . y_{5}, X \neq Y ; 5$ days $D=d_{1} . . d_{5}, D^{\prime}=d_{1} . . d_{5}, D^{\prime \prime}=d_{1} . . d_{5} ;$ and 8 tasks $T=t_{1} . . t_{8}, T^{\prime}=t_{1} . . t_{8}, T^{\prime \prime}=t_{1} . . t_{8}$.

The first curve shows a very low EER (2.8\%) when the reference template is day and task specific, and the genuine/impostor template come from recordings of the same day and task: $\operatorname{DET}\left(T_{X, d_{i}, t_{j}}^{R}, T_{X, d_{i}, t_{j}}^{G}, T_{Y, d_{i}, t_{j}}^{I}\right)$ with $i \in 1 . .5$ and $j \in 1$..8. The second DET curve shows the $4 \mathrm{~km} / \mathrm{h}$ walking speed specific performance (weight and shoe independent). The reference template combines the 5 tasks with $4 \mathrm{~km} / \mathrm{h}: \operatorname{DET}\left(T_{X, d_{i}, T_{4 k m / h}}^{R}, T_{X, d_{i}, T_{4 k m / h}}^{G}, T_{Y, d_{i}, T_{4 k m / h}}^{I}\right)$, with $i \in 1 . .5$ and $T_{4 k m / h}=t_{1}, t_{2}, t_{4}, t_{7}, t_{8}$. The third DET curve shows the task-independent performance. The reference template is day dependent but combines all 8 tasks: 
$\operatorname{DET}\left(T_{X, d_{i},{ }_{T}}^{R}, T_{X, d_{i}, T^{\prime}}^{G}, T_{Y, d_{i}, T^{\prime \prime}}^{I}\right)$. The more the template is task-independent, the lesser the authentication performance, due to the template being less specific to a particular task. The fourth DET curve with $\operatorname{DET}\left(T_{X, D, t_{j}}^{R}, T_{X, D^{\prime}, t_{j}}^{G}, T_{Y, D^{\prime \prime}, t_{j}}^{I}\right)$ shows the day-independent performance. Performance is lower than with daydependent templates, which implies that day to day changes affect the gate much in the same way as tasks affect the gait, making it difficult to capture in a day-independent reference template. As expected the authentication performance decreases with decreasing MOS difference. Comparing the MOS values of figure $3 \mathrm{a}$ ) with the MOS values of figure 3 b) there is a large difference, therefore the EER is very low (2.8\%). The MOS values of the first 4 bars in figure 3 c) are closer to the MOS values of figure $3 \mathrm{~b}$ ) than to the values of figure $3 \mathrm{a}$ ). This gives a higher EER of $17.6 \%$ (second curve in fig 5 . Including the different speed lowers the distance between genuine trials and impostor trials even more, resulting in the worst EER of $31.5 \%$ (third curve in fig [5).

\section{Conclusion}

We analyzed biometric user authentication in real-world scenarios using gait acceleration signals. We introduced a statistical measure which we called Measure Of Similarity (MOS) to characterize the similarity of two gait data sets. Using this MOS value we showed that variations due to weight, shoes, speed and day to day variability reduces the distance between genuine and impostor data sets, making the gait-based authentication in real-life environments challenging. In particular we showed that walking speed changes the nature of the gait acceleration profile and cannot be compensated by frequency and amplitude normalization. Change in shoes also strongly affects the gait acceleration profile. A change in weight has less effect on the acceleration profile, and can be minimized by normalization. We evidenced large day to day gait variations. Longer recordings are required to assess whether this was due to users learning to walk on the treadmill or due to natural variability. In the latter case this may question the real-world applicability of gait-based authentication using the simple features used here. Actual user authentication supports the results of the statistical similarity analysis. Authentication thus must be performed in conditions similar to those of training.

Improvements to gait-based authentication include analyzing the gait only when the actual walking conditions match that of training. This may be easily applied to walking speed and weight (e.g. with pressure sensitive floors). Several training sets may be collected (e.g. various speeds) and used accordingly automatically. The new statistical measure of similarity allows to identify the feature vector components with the highest information content (e.g. regions of the acceleration profile or set of frequency components). Giving more weight to these components when matching a feature vector to a template may better results. Finally, since the gait acceleration profile is affected despite normalization by walking speed, shoes or weight, new features beyond simple acceleration profile or frequency components must be investigated that better capture the unique characteristics of gait. 
Finally we note that gait analysis may be used to identify the user's context, which is especially important in the field of pervasive computing and wearable computing 8. Our results show that the gait contains information beyond walking speed, in particular about the weight that a person carries or the shoes that are worn. This information may be automatically interpreted and presents an opportunity for gait-based context-aware systems, as a complement to other sensors used in context-aware systems. Application scenarios include smart devices or clothing that adapt to their user.

Acknowledgment. This project is funded by the EU projects DAPHNet, contract number: 018474-2 (www.daphnet.eu) and SEAT, contract number: 030958 (www.seat-project.org). All views here reflect the authors' opinion and not that of the commission.

\section{References}

1. Ailisto, H., Lindholm, M., Mäntyjärvi, J., Vildjiounaite, E., Mäkelä, S.-M.: Identifying people from gait patterns with accelerometers. Proceedings of Biometric Technology for Human Identification II 5779, 7-14 (2005)

2. Bächlin, M., Roggen, D., Tröster, G.: Context-aware platform for long-term life style management and medical signal analysis. In: Proceeding of the 2nd Sensation International Conference, Chania, Greece (2007)

3. Snekkenes, E., Gafurov, D., Buvarp, T.E.: Robustness of biometric gait authentication against impersonation attack. In: 1st Int. Workshop on Information Security, On The Move Federated Conferences, France (2006)

4. Gafurov, D., Snekkenes, E., Bours, P.: Spoof attacks on gait authentication system. IEEE Trans. on Information Forensics and Security 2(3), 491-502 (2007)

5. Gafurov, D., Helkala, K., Søndrol, T.: Biometric gait authentication using accelerometer sensor. Journal of computers 1(7), 51-59 (2006)

6. Gafurov, D., Helkala, K., Sondrol, T.: Gait recognition using acceleration from mems. In: Proc. of 1st Int. Conf. on Availability, Reliability and Security, USA, pp. 432-439 (2006)

7. Han, J., Bhanu, B.: Individual recognition using gait energy image. IEEE Transactions on Pattern Analysis and Machine Intelligence 28(2), 316-322 (2006)

8. Mann, S.: Wearable computing as means for personal empowerment. In: 1st Int. Conf. on Wearable Computing. IEEE Computer Society Press, Los Alamitos (1998)

9. Mäntyjärvi, J., Lindholm, M., Vildjiounaite, E., Makela, S.-M., Ailisto, H.A.: Identifying users of portable devices from gait pattern with accelerometers. In: Int. Conf. on Acoustics, Speech, and Signal Processing, vol. 2, pp. 973-976 (2005)

10. Rong, L., Zhiguo, D., Jianzhong, Z., Ming, L.: Identification of individual walking patterns using gait acceleration. In: 1st Int. Conf. on Bioinformatics and Biomedical Engineering, pp. 543-546 (2007)

11. Sarkar, S., Phillips, P.J., Liu, Z., Robledo, I., Grother, P., Bowyer, K.W.: The human id gait challenge problem: Data sets, performance, and analysis. IEEE Transactions on Pattern Analysis and Machine Intelligence 27(2), 162-177 (2005)

12. Vildjiounaite, E., Mäkelä, S.-M., Lindholm, M., Riihimäki, R., Kyllönen, V., Mäntyjärvi, J., Ailisto, H.: Unobtrusive multimodal biometrics for ensuring privacy and information security with personal devices. In: Fishkin, K.P., Schiele, B., Nixon, P., Quigley, A. (eds.) PERVASIVE 2006. LNCS, vol. 3968, pp. 187-201. Springer, Heidelberg (2006) 